\title{
A Mass Zero Cluster Expansion
}

\author{
Part 1. The Expansion * \\ Paul G. Federbush \\ Department of Mathematics, The University of Michigan, Ann Arbor, MI 48109, USA
}

\begin{abstract}
A cluster expansion is developed and applied to study the perturbation $\lambda(\Delta \phi)^{4}$ of the massless lattice field $\phi$ in dimension 3 . The method is loosely inspired by the work of Gawedzki and Kupiainen on block spin techniques for the $\lambda(\vec{\nabla} \phi)^{4}$ system. The cluster expansion is given in terms of expansion coefficients for the field as a sum of certain special block spin functions. These functions are chosen with a large number of moments zero, so that the interaction couples spatially separated functions with an interaction falling off as a high inverse power of the separation distance. The present techniques, with some technical development, should work for broad classes of other models, including the lattice dipole gas and the $\lambda(\vec{\nabla} \phi)^{4}$ model. Models $\lambda\left(|\Delta|^{\alpha} \phi\right)^{2 s}, \alpha>\frac{1}{2}$, are essentially included in the present work.
\end{abstract}

\section{Introduction}

Cluster expansions have had a wide variety of applications in field theory and statistical mechanics. They have been used to prove the existence of correlation functions in the thermodynamic limit, properties of the spectrum, clustering of correlation functions, the existence of phase transitions, Debye screening, and many other detailed properties of very diverse systems. Typically they may be applied to systems where such special techniques as correlation inequalities, reflection positivity, and Markov properties may not apply. It is of obvious interest to extend the applicability of cluster techniques to systems with zero masses - systems with long range interactions.

In this paper we develop a new cluster expansion and apply it to the lattice model with Hamiltonian density $\frac{1}{2}(\nabla \phi)^{2}+\lambda(\Delta \phi)^{4}$ in 3 dimensions. Other models, such as the lattice dipole gas (or continuum dipole gas) or the $\lambda(\vec{\nabla} \phi)^{4}$ model, should be accessible to similar treatment with increased technical difficulties. We intend to come back to study the dipole gas in a later paper. We do not know if the present techniques will be useful in studying models such as the rotator model or the Heisenberg model. Part 1 of this paper introduces the general concepts, defines the cluster expansion, and contains basic estimates, all of which should be useful in further applications. Part 2 contains the combinatoric details involved in proving the convergence of the cluster expansion.

* This work was supported in part by the National Science Foundation under Grant No. PHY 79-05688 
Our expansion was inspired in a general way by the work of Gawedzki and Kupiainen [3] and Gallavotti et al. [1], who rigorized earlier ideas of physicists, particularly Wilson and Kadanoff (see [3] for further references), all using the idea of block spins. We introduce (in Sect. 4) an orthonormal set of functions, $\psi_{k}(x)$, on the lattice, and define our block spin variables as

$$
\alpha_{k}=\sum_{x}\left(\sqrt{-\Delta} \psi_{k}\right)(x) \phi(x)
$$

The $\psi_{k}(x)$ live on cubes of various sizes. The basic trick we employ is constructing the $\psi_{k}(x)$ with a certain large number of their moments equal zero (see Sect. 3). This easily results in the fact that the interaction couples $\alpha_{k_{1}}$ and $\alpha_{k_{2}}$ with a strength that falls off as a high inverse power of the distance between the cubes to which $\alpha_{k_{1}}$ and $\alpha_{k_{2}}$ are associated (Sect. 7), via the interaction term $\lambda(\Delta \phi)^{4}$.

Successive terms in the cluster expansion defined in Sect. 8, involve coupling more and more $\alpha_{k}$, rather than larger and larger regions of space as is the usual situation. The basic physical idea that leads to convergence is that modes that couple over large distances are favored to have large scale structure; the small scale modes have weak coupling at large distance (with the conditions on moments we have enforced defining the modes). The usual cluster expansions do not take advantage of this distinction between small scale and large scale modes.

In the development of the cluster expansion a device involving "tadpoles" is introduced. This requires any subset of small scale variables to couple at least twice with large scale variables with which it interacts. A detailed explanation of this process is given in Sect. 8. It is the reflection of the renormalization group philosophy-integrating out first the smallest scale (highest momentum) variables and then proceeding to the next scale iteratively-as it appears in the present procedure.

We point out that the details of the present paper would remain virtually unchanged in treating a model with interaction $\lambda\left(|\Delta|^{\alpha} \phi\right)^{4}$ for $\alpha>\frac{1}{2}$. (Although such models have an effective interaction that is integrable, the usual cluster expansion will not work unless $\alpha$ is quite large because of the number divergence.) In Sect. 8.F we discuss the two point correlation function for these models.

There is a growing body of interesting papers on subjects related to the problem of the present paper. We do not attempt a complete catalogue of papers and principal results. The work of Gawedzki and Kupiainen in [3] derives asymptotic expansions for thermodynamic functions in the $\lambda(\vec{\nabla} \phi)^{4}$ model. The work of Bricmont, Fontaine, Lebowitz, and Spencer in [7] derives, in addition, asymptotic expansions for correlation functions in the same model. The work of Fröhlich and Spencer in [5] derives some estimates for two point clustering in a hard core dipole gas. Malyshev and Tirozzi in [8] prove a cluster expansion for the models considered in this paper.

We could have tried to use expansion functions developed in [3] instead of the $\psi_{k}$ given in Sect. 4. We feel our functions have some advantages; in particular we can handle in the present paper models $\lambda \prod_{i}\left(|\Delta|^{\alpha_{i}} \phi\right)^{2 s_{i}}, \alpha_{i}>1 / 2$, with no essential changes, but $I$ believe this would not be possible using the functions in [3]. We are not sure whether the technique in [8] applies to this same set of models. 
The principal advantage of our cluster expansion over that in [8], however, is that our technique should extend to $\lambda(\vec{\nabla} \phi)^{4}$ and the dipole gas. In [6] Gawedzki has tested, successfully, some of the critical ideas that will be involved in extending the present work to the $\lambda(\vec{\nabla} \phi)^{4}$ and dipole gas situation. The study of clustering properties in mass zero situations is a field of great importance and growing interest; it will assume form by the cumulative efforts of many researchers.

\section{The System}

We consider a lattice model with Hamiltonian density $\frac{1}{2}(\nabla \phi)^{2}+\lambda(\Delta \phi)^{4}$. The lattice is a cubical lattice, with unit spacing, $p^{N}$ sites along each edge; thus $\left(p^{N}\right)^{3}$ sites in total. We may consider this a subset, $A$, of the lattice $Z^{3}$ in $R^{3}$ of points with integral coordinates. $p$ is a fixed integral parameter; the lattice size becomes arbitrarily large as $N$ increases, $N$ an integer determining the volume of the lattice. (The infinite volume limit, $A \rightarrow \infty$, becomes the limit $N \rightarrow \infty$.)

The partition function may be written

$$
Z=\prod_{x \in A}\left(\int d \phi_{x}\right) e^{-\sum_{x \in A}\left((1 / 2)(\vec{\nabla} \phi)_{x}^{2}+\lambda(\Delta \phi)_{x}^{4}\right)} \delta\left(\sum_{x \in A} \phi_{x}\right) .
$$

$\phi$ at the site $x$ is written as $\phi_{x}$ or $\phi(x)$, similarly with other functions on the lattice. The $\phi_{x}$ are determined for all $x$ in $Z^{3}$ by periodicity, a change in any coordinate by $p^{N}$ leaving $\phi$ invariant. This leads to periodic boundary conditions on the Laplacian. (We have handled the zero frequency mode of the periodic Laplacian with the $\delta$ function.) We let $[\mathscr{A}(\phi)]$, for $\mathscr{A}(\phi)$ a function of the $\phi_{x}$, be the same integral as in (2.1), but with $\mathscr{A}(\phi)$ added to the integrand. We also set

$$
\langle\mathscr{A}(\phi)\rangle=\frac{1}{Z}[\mathscr{A}(\phi)] \text {. }
$$

\section{Moments}

We work with the unit lattice, $Z^{n}$, in $R^{n}(n=3$ plays no special role in this section) of points with integral coordinates. $\Delta_{i}, i=1, \ldots, n$ are the difference operators, obvious generalizations of $\Delta$ in one dimension, that satisfies $(\Delta f)_{i}=f(i+1)-f(i)$. We have higher difference operators

$$
\Delta_{\alpha}=\left(\Delta_{1}\right)^{\alpha_{1}} \ldots\left(\Delta_{n}\right)^{\alpha_{n}},|\alpha|=\Sigma \alpha_{i},
$$

and similarly monomials

$$
x^{\alpha}=\left(x_{1}\right)^{\alpha_{1}} \ldots\left(x_{n}\right)^{\alpha_{n}}
$$

The basic relation

$$
\left(\Delta_{i}(f g)\right)_{x}=\left(\left(\Delta_{i} f\right) g\right)_{x}+\left(\left(\Delta_{i} g\right) f\right)_{x}+\left(\left(\Delta_{i} f\right)\left(\Delta_{i} g\right)\right)_{x}
$$

holds, here $x$ is a lattice site. We write

$$
\int f=\sum_{x} f_{x}
$$


the sum over lattice sites. We let $\mathscr{D}$ be a finite connected subset of the lattice (one may go from any point in $\mathscr{D}$ to any other via a sequence of points in $\mathscr{D}$, nearest neighbors in the original lattice). $f$ is supported in $\mathscr{D}$ if $f=0$ on all points not in $\mathscr{D}$. We collect some simple results on moments, with a sketch of proofs.

Theorem 3.1. If $f=\sum_{i} \Delta_{i} g^{(i)}$ where each $g^{(i)}$ has finite support, then $\int f=0$. Theorem 3.2. If $f=\sum_{|\alpha|=s+1} \Delta_{\alpha} g^{(\alpha)}$, where each $g^{(\alpha)}$ has finite support, then $\int f x^{\alpha}=0$
for $|\alpha| \leqq s$.

Theorem 3.3. If $f$ has support in $\mathscr{D}$, and $\int f=0$, then there are $g^{(i)}(i=1,2, \ldots, n)$ supported in $\mathscr{D}$ with $f=\sum_{i} A_{i} g^{(i)}$.

Theorem 3.4. If $f$ has support in $\mathscr{D}$, and $\int f x^{\alpha}=0,|\alpha| \leqq s$, then there are $g^{(\alpha)},|\alpha|=$ $s+1$, supported in $\mathscr{D}$ with $f=\sum_{|\alpha|=s+1} \Delta_{\alpha} g^{(\alpha)}$.

Theorem 3.5. If $\mathscr{D}$ is a cube of side $L$ ( $L$ vertices on an edge) there are constants $c_{s}$ (independent of $L$ ) such that the $g^{(\alpha)}$ of Theorem 3.4 may be picked satisfying

$$
\left|g^{(\alpha)}\right|_{\infty} \leqq c_{s} L^{s+1}|f|_{\infty}
$$

If $g$ has finite support $\int \Delta_{i} g=0$. This is all Theorem 3.1 says.

Theorem 3.2 is proved combining Theorem 3.1 with the integration by parts formula (3.3). The last term in (3.3) does not cause difficulty.

Preparatory to proving Theorem 3.3 and 3.4 we note that in $R^{1}$ if $f(k)=1$, $f(k+1)=-1$, and $f(i)=0, i \neq k$ or $k+1$; then $f=\Delta g$ with $g(k+1)=1$ and $g(i)=0, i \neq k+1$. Similarly in $R^{n}$, a function $f$ zero except for equal and opposite values at two nearest neighbor lattice sites - we call this an "elementary" functioncan be written as $\Delta_{j} g$ with $j$ the direction parallel to the bond joining the two points, and $g=0$ except at one of the two points in which $f \neq 0$. But any $f$, as in Theorem 3.3, is a finite sum of such "elementary" functions, and this proves Theorem 3.3. Theorem 3.4 is proven from Theorem 3.3 inductively using integration by parts ((3.3) and Theorem 3.1). We illustrate the first few steps.

$$
\begin{aligned}
\int f=0 & \Rightarrow f=\sum_{i} \Delta_{i} g_{1}^{(i)} \\
\int x_{k} f=0 & \Rightarrow \int x_{k}\left(\sum_{i} \Delta_{i} g_{1}^{(i)}\right)=0 \\
& \Rightarrow \int g_{1}^{(k)}=0 \\
& \Rightarrow g_{1}^{(k)}=\sum_{i} \Delta_{i} g_{2, k}^{(i)}, \text { etc. }
\end{aligned}
$$

Viewing the inductive procedure to prove Theorem 3.4, we may observe it is sufficient to prove Theorem 3.5 for $s=0$. We prove this $s=0$ case inductively on the dimension $n$. With

$$
f=f\left(x_{1}, \ldots, x_{n}\right)
$$


we define

$$
\bar{f}\left(x_{1}, \ldots, x_{n}\right)=\frac{1}{L^{n-1}} \sum_{\bar{x}_{1}, \ldots, \bar{x}_{n-1}} f\left(\bar{x}_{1}, \ldots, \bar{x}_{n-1}, x_{n}\right)
$$

inside the cube and zero outside the cube. We write

$$
f=\bar{f}+(f-\bar{f}) \text {. }
$$

It is trivial that

$$
\bar{f}=\Delta_{n} g^{(n)}
$$

with

$$
\left|g^{(n)}\right|_{\infty} \leqq L|\bar{f}|_{\infty} \leqq L|f|_{\infty}
$$

and $g^{(n)}$ living in the cube. This is a one-dimensional problem. $(f-\bar{f})$ may be written as

$$
(f-\bar{f})=\sum_{i=1}^{n-1} \Delta_{i} g^{(i)} .
$$

This involves solving $(n-1)$ dimensional problems only. We have used

$$
\int d \bar{x}_{n} \bar{f}\left(x_{1}, \ldots, \bar{x}_{n}\right)=0
$$

and

$$
\int d \bar{x}_{1}, \ldots, d \bar{x}_{n-1}(f-\bar{f})\left(\bar{x}_{1}, \ldots, \bar{x}_{n-1}, x_{n}\right)=0 .
$$

The estimate (3.4) may be chosen to hold for the $g^{(i)}$ (with $s=0$ ) by the inductive hypothesis. Note that

$$
|f-\vec{f}|_{\infty} \leqq 2|f|_{\infty}
$$

\section{Expansion Functions $\psi_{k}$}

In this section we construct the expansion functions $\left\{\psi_{k}\right\}$; these will form a complete orthonormal set of functions supported on the lattice $A$. Thus there are $\left(p^{N}\right)^{3}$ such functions. $M$ and $s$, integers (each determining the other) are fixed parameters of our whole procedure, where there are $M$ linearly independent polynomials of degree less than or equal to $s$. All but $M$ of the functions $\psi_{k}$ will satisfy

$$
\int \psi x^{\alpha}=0|\alpha| \leqq s
$$

Those $\psi_{k}$ satisfying (4.1) will be said to have zero "first $M$ moments."

The index $k$ on $\psi_{k}$ is viewed as a triple label, $k=(r, \gamma, t)=\left(r_{k}, \gamma_{k}, t_{k}\right), r$ is an integer, $1 \leqq r \leqq N$. The support of $\psi_{k}$ is a cube of size $\left(p^{r}\right)^{3}$. (There are $p^{r}$ sites on its edge.) $y$ labels which one of the $\left(p^{N}\right)^{3} /\left(p^{r}\right)^{3}$ locations for the support of $\psi_{k}$, that $\psi_{k}$ lives on. (The supports of the various $\psi_{k}$, with fixed $r$ value, form a lattice.)

We will require that the "first $M$ moments" of all the $\psi_{k}$ with $r$ values less 
than $N$ are zero. (We assume for convenience that the $M$ monomials $x^{\alpha}$, with $|\alpha| \leqq s$, are linearly independent when restricted to a cube of size $p^{3}$, i.e. $p$ vertices on an edge. This requires for fixed $M$ that $p$ be sufficiently large.) This requirement guides the construction of the $\psi_{k}$. For fixed $r$ and $\gamma$, the number of possible $t$ values are

$$
\#\{t\}=\left\{\begin{array}{cl}
M p^{3}-M & r=2, \ldots, N-1 \\
p^{3}-M & r=1 \\
M p^{3} & r=N .
\end{array}\right.
$$

It is easy to count that the total number of $\psi_{k}$ is $\left(p^{N}\right)^{3}$. One may construct the $\psi_{k}$ inductively on the value of $r . \psi_{k}$, for a given $r$ value, $r_{k}$, must be orthogonal to all the $\psi_{k}$ with $r$ value less than $r_{k}$. If the $r$ value is less than $N, \psi_{k}$ must also have zero "first $M$ moments." We will proceed to find a more explicit description of the $\psi_{k}$.

We first consider the $r=1$ case. $\psi_{k}$ for fixed $\gamma$ lives on a cube of size $p^{3}$. We require the $M$ conditions (4.1) to hold for the functions we keep. Clearly a complete orthonormal set of functions satisfying (4.1) living on this cube has dimension $\left(p^{3}-M\right)$. These $p^{3}-M$ functions (an arbitrary complete orthonormal set) are labelled by $t$.

For $r>1$ we divide the $\left(p^{r}\right)^{3}$ size cube into $p^{3}$ subcubes of size $\left(p^{r-1}\right)^{3}$, by $3(p-1)$ division planes $((p-1)$ planes perpendicular to each coordinate axis, equally spaced). On each subcube $\psi_{k}$ is of the form

$$
\sum_{|\alpha| \leq s} a_{\alpha} x^{\alpha}
$$

On each subcube this is a vector space of dimension $M$. For the $r=N$ case no further condition is enforced. The total dimensionality of the vector space is $M p^{3}$. The $t$ labels a basis for this space, arbitrarily chosen except that we require one of the elements in the basis to be the constant function, the zero frequency mode.

For the case $1<r<N$, on the vector space of dimension $M p^{3}$, one is restricted to functions satisfying (4.1). The $t$ labels an arbitrarily chosen basis for this space, of dimension $M p^{3}-M$; but at the end of this section we present some natural conditions on the choice of bases.

The reader should convince himself that the $\psi_{k}$ corresponding to different values of $(r, \gamma)$ are automatically orthogonal by this construction!

We proceed to give some useful estimates on the $\psi_{k}$. We will write $p^{r}=p^{r_{k}}$ $=L=L_{k}$, using $L$ to denote the edge length of the cube on which $\psi_{k}$ is supported. We use the standard notation of $c$ for positive constants, with possibly different values in different equations.

Estimate 4.1. $\left|\psi_{k}\right|_{\infty} \leqq \frac{c}{L^{3 / 2}}$.

Combining Estimate 4.1 with Theorem 3.5 we get

Estimate 4.2. If $r_{k}<N$ and $\bar{s} \leqq s+1$, then we may write

$$
\psi_{k}=\sum_{|\alpha|=\bar{s}} \Delta_{\alpha} \psi_{k}^{(\alpha)}
$$


with the $\psi_{k}^{(\alpha)}$ supported on the cube $\left(r_{k}, \gamma_{k}\right)$ and satisfying

$$
\left|\psi_{k}^{(\alpha)}\right|_{\infty} \leqq L^{\bar{s}} \frac{c}{L^{3 / 2}} .
$$

Estimate 4.3. We consider $\Delta_{i} \psi_{k}$. Away from the $(p-1)$ division planes perpendicular to the $i$-axis and the two boundary planes perpendicular to the $i$-axis (for a plane $x_{i}=j+\frac{1}{2}$, the sites with $x_{i}=j$ or $j+1$ are excluded), one has

$$
\left|\Delta_{i} \psi_{k}\right|_{\infty} \leqq \frac{1}{L} \frac{c}{L^{3 / 2}} .
$$

Along the division and boundary planes (at the above excluded sites) one only assumes

$$
\left|\Delta_{i} \psi_{k}\right|_{\infty} \leqq \frac{c}{L^{3 / 2}} .
$$

We observe that the conditions (4.1) are translation invariant. Thus we may choose the $\psi_{k}$ such that for fixed values of $r_{k}$ and $t_{k}$, the $\psi_{k}$ (as $\gamma_{k}$ varies) are translates of one another. Consider the set of real functions on $[0, p]^{3}$, with the property that when restricted to the interior of each unit cube with integral vertices in $[0, p]^{3}$, they are polynomials of degree $\leqq s$. Impose the additional conditions

$$
\int_{[0, p]^{3}} \psi x^{\alpha} d^{3} x=0 \quad|\alpha| \leqq s
$$

for all $\psi$ in this set, where here the integral is with respect to the usual Lebesgue measure on $R^{3}$. This set of functions, viewed as elements of $L^{2}\left([0, p]^{3}, \mu\right)$, is $M p^{3}$ $-M$ dimensional. ( $\mu$ is the restriction of ordinary Lebesgue measure on $R^{3}$.) Let $\left\{\phi_{\alpha}(x)\right\}$ be a basis for this subset of $L^{2}\left([0, p]^{3}, \mu\right), \alpha=1,2, \ldots, M p^{3}-M$. The functions $\left\{\psi_{k}(x)\right\}, 1<r_{k}<N$, may be chosen, approximately, as scaled, translated, discretized versions of the $\phi_{\alpha}(x)$

$$
\psi_{k}(x) \cong \frac{1}{\left(p^{r_{k}-1}\right)^{3 / 2}} \phi_{t_{k}}\left(\frac{x-R\left(\gamma_{k}\right)}{p^{r_{k}-1}}\right) .
$$

Here $R\left(\gamma_{k}\right)$ is the translation. This indicates an approximate scaling symmetry of the formalism, useful for applications of renormalization group theory. (4.9) may be arranged to be a "better approximation" the larger $r_{k}$ becomes. Estimates 4.1 and 4.3 are thus easily understood.

Treating the considerations of the last paragraph more quantitatively, suppose $\omega$ is any of the $p^{3}$ unit cubes in $[0, p]^{3}$, and that $\frac{x-R\left(\gamma_{k}\right)}{p^{r_{k}}-1}$ is within this cube. Writing the right side of (4.9) as

$$
\sum_{|\alpha| \leqq s} \frac{1}{\left(p^{r_{k}}\right)^{3 / 2}} A\left(\alpha, \omega, t_{k}\right)\left(\frac{x-R\left(\gamma_{k}\right)}{p^{r_{k}-1}}\right)^{\alpha}
$$

and the left side as

$$
\sum_{|\alpha| \leqq s} \frac{1}{\left(p^{r_{k}}\right)^{3 / 2}} \tilde{A}\left(\alpha, \omega, t_{k}, r_{k}\right)\left(\frac{x-R\left(\gamma_{k}\right)}{p^{r_{k}-1}}\right)^{\alpha}
$$


we assert one may choose the $\psi_{k}(x)$ so that

$$
\left|A\left(\alpha, \omega, t_{k}\right)-\tilde{A}\left(\alpha, \omega, t_{k}, r_{k}\right)\right| \underset{r_{k} \rightarrow \infty}{\longrightarrow} 0
$$

for all $t_{k}, \omega, \alpha$ (for all $\left(M p^{3}-M\right) p^{3} M$ of these quantities). This of course is a stronger result than we need in Estimates 4.1 and 4.3, or anywhere else in this paper.

We note the following elementary matrix lemma.

Lemma 4.4. In a $D$ dimensional inner product space there are constants $c_{1}(D), c_{2}(D)$ such that for any set of $D$ vectors $v_{1}, \ldots, v_{D}$ satisfying

$$
\left\langle v_{i}, v_{j}\right\rangle=\delta_{i j}+\varepsilon_{i j}
$$

with

$$
\left|\varepsilon_{i j}\right| \leqq c_{1}(D), \quad \text { all } i, j,
$$

there are $L_{i j}$ satisfying

$$
\left|L_{i j}\right| \leqq c_{2}(D) \operatorname{Sup}\left|\varepsilon_{i j}\right|
$$

such that the vectors $w_{i}$

$$
w_{i}=v_{i}+\sum_{j} L_{i j} v_{j}
$$

from an orthonormal basis.

This lemma may be applied in our situation, with $\left\{v_{i}\right\}$ the vectors on the right side of (4.9), the approximations to the $\left(\psi_{k}\right)$. The $\left|\varepsilon_{i j}\right| \underset{r_{k} \rightarrow \infty}{\stackrel{2}{\longrightarrow}} 0$, just as Riemann sum approximations to $\int_{\{0, p]} \phi_{t_{1}}(x) \phi_{t_{2}}(x) d^{3} x$ approach the integral.

Perhaps we should add that Estimates 4.1-4.3 do not depend on choosing $\psi_{k}$ to have scaling and translation properties.

\section{The Change of Field Variables}

We now change variables from $\phi(x), x \in A$, to the expansion coefficients of $\phi$ in terms of the $\psi_{k}$. That is, we write

$$
\phi(x)=\sum_{k} \alpha_{k}\left(\frac{1}{\sqrt{-4}} \psi_{k}\right)(x) .
$$

The $\psi_{k}(x)$ are numerical functions, defined in the last section, and the $\alpha_{k}$ are the new variables. Since the $\psi_{k}(x)$ are orthonormal we also have

$$
\alpha_{k}=\sum_{x}\left(\sqrt{-\Delta} \psi_{k}\right)(x) \phi(x)
$$

The $\alpha_{k}$ are only approximately localized in space; $\alpha_{k}$ depends on the variables $\phi(x)$ roughly over a cube of size $\left(p_{k}^{r}\right)^{3}$.

The cluster expansion we will develop will be patterned (with some crucial differences) on previous cluster expansions, but involving the interaction of larger and larger subsets of the $\alpha_{k}$. 


\section{The New Free Covariance}

We define as usual the free covariance of the $\phi(x)$ as

$$
C(x, y)=\langle\phi(x) \phi(y)\rangle_{0}=-\frac{1}{4},
$$

where the subscript 0 indicates the expectation is taken with $\lambda=0$. Similarly we define

$$
C\left(k, k^{\prime}\right)=\left\langle\alpha_{k} \alpha_{k^{\prime}}\right\rangle_{0}
$$

(using the same notation for the covariance in terms of the new variables). From equation (5.2) we clearly have

$$
\begin{aligned}
C\left(k, k^{\prime}\right) & =\int d x \int d y\left(\sqrt{-\Delta} \psi_{k}\right)(x)\langle\phi(x) \phi(y)\rangle_{0}\left(\sqrt{-\Delta} \psi_{k^{\prime}}\right)(y) \\
& =\delta_{k, k^{\prime}}
\end{aligned}
$$

The new covariance is simple as could be! (The single mode which is a zero eigenvector of the Laplacian never causes us difficulties.)

\section{Interaction Estimates}

To study the interaction $\lambda(\Delta \phi)^{4}$, we need estimates for $\left(\Delta \frac{1}{\sqrt{-\Delta}} \psi_{k}\right)(x)$. We will state our results, for simplicity, in the case when the Laplacian is the infinite volume operator. It is not challenging to derive estimates for the periodic case from these results. The basic ingredients are estimates for the kernels $\left(\Delta_{\alpha} \frac{1}{\sqrt{-\Delta}}\right)$,

$$
\left|\left(\Delta_{\alpha} \frac{1}{\sqrt{-\Delta}}\right)_{x y}\right| \leqq \frac{c_{\alpha}}{|x-y|^{2+|\alpha|}} .
$$

We also use the translation invariance of these kernels and Estimates 4.2-4.3.

Corresponding to $\psi_{k}, k \leftrightarrow\left(r_{k}, \gamma_{k}, t_{k}\right)$, there is the cube $\left(r_{k}, \gamma_{k}\right)$ of side length $L=L_{k}=(p)^{r_{k}}$. Let $\hat{\partial}\left(r_{k}, \gamma_{k}\right)$ be defined as the union of the boundary planes of the cube, with the portions of its division planes interior to it (a union of $p^{3}$ hollow boxes). We let $\hat{d}(x)$ be defined as

$$
\hat{d}_{k}(x)=\hat{d}(x)=\max \left\{1, \operatorname{dist}\left(x, \hat{\partial}\left(r_{k}, \gamma_{k}\right)\right)\right\}
$$

for a lattice point $x$.

Estimate 7.1. If $\hat{d}(x) \geqq L$ and $r_{k}<N$ then

$$
\left|\left(\Delta \frac{1}{\sqrt{-4}} \psi_{k}\right)(x)\right| \leqq \frac{c L^{s+5 / 2}}{(\hat{d})^{5+s}}=c\left(\frac{L}{\hat{d}}\right)^{5+s} \frac{1}{L^{5 / 2}} .
$$

Estimate 7.2. If $\hat{d}(x) \leqq L$ then

$$
\left|\left(\Delta \frac{1}{\sqrt{-\Delta}} \psi_{k}\right)(x)\right| \leqq \frac{c}{L^{5 / 2}} \ln L+\frac{c}{L^{3 / 2}} \frac{1}{d} .
$$


If $r_{k}=N$, and $\psi_{k}$ is not the zero frequency mode, (7.4) also holds with $\Delta$ replaced by the periodic Laplacian.

Estimate 7.1 arises from Estimate 4.2 and (7.1) upon integrating by parts the derivatives in Estimate 4.2 onto the kernel. Estimate 7.2 is derived by passing one difference in the Laplacian onto $\psi_{k}$, and using Estimate 4.3. The first term in the right side of (7.4) arises from (4.6), and the second term from (4.7).

\section{The Cluster Expansion}

We will now derive a cluster expansion for $[\mathscr{A}], \mathscr{A}$ a polynomial in a finite number of the $\alpha_{k}$, the distinguished variables. We will have

$$
[\mathscr{A}]=\sum_{T}[\mathscr{A}]_{T}=\sum_{T} K_{T}(\mathscr{A}) Z^{T^{c}}
$$

The T's label terms in the cluster expansion. As our starting expression we have

$$
[\mathscr{A}]=\prod_{k}\left(\int d \alpha_{k}\right) \mathscr{A}(\alpha) e^{-(1 / 2) \sum_{k} \alpha_{k}^{2}} e^{-\lambda \sum_{x}(\Delta \phi)_{x}^{4}}
$$

$Z$ is the same expression with $\mathscr{A}=1$. We have changed the normalization of $Z$ by a harmless numerical factor. In $(8.2)(\Delta \phi)^{4}$ is understood expressed in terms of the $\alpha$ variables. In the definition of the cluster expansion, we will first give a complex formulation of the labelling of terms and then an iterative construction of each term.

The classic paper of Glimm, Jaffe and Spencer [4] is the source of the basic constructs in a cluster expansion, [2] is also a source of some of our ideas.

\section{A. Spatial Sequence Interpolation}

We select an arbitrary but fixed linear ordering on the $\left(p^{3}\right)^{N}$ lattice sites. $(i, x)$ is an ordered pair consisting of a strictly positive integer, $i$, and a lattice site, $x$. On such pairs we establish the linear ordering

$$
(i, x)>(j, y) \text { if } \begin{cases}\text { either } & i>j \\ \text { or } & i=j \text { and } x>y .\end{cases}
$$

We let $G_{0}$ be the set of all possible selections of four $\alpha$ 's (non-ordered and allowing repetitions). $G=N \cup G_{0}, N$ a symbol we are now introducing. (In a schematized step in the interpolation

$$
e^{v(1)}=e^{v(0)}+\int_{0}^{1} \frac{d}{d s} e^{v(s)} d s
$$

The element $N$ in $G$ will correspond to the $e^{v(0)}$ term; and the selection of four $\alpha$ 's will correspond to a term in which a monomial specified by these four $\alpha$ 's is differentiated from the exponent.)

The labelling symbol $T$ in (8.1) is a mapping from the space of pairs $(i, x)$ into $G$, that is eventually into $N$, i.e., for any fixed $T$, if $(i, x)$ is large enough (in the ordering (8.A.1)), $T:(i, x) \rightarrow N$. Many of the $T$ 's will in fact correspond to terms identically zero. (By putting further conditions on the mapping $T$ one could eliminate such zero terms.) 
The interaction $\lambda \sum_{x}(\Delta \phi)_{x}^{4}$ is a sum of contributions from the different lattice sites. As the labelling indicates, and as we will see in the next section, interpolation occurs in each of these terms separately! Somehow this seems to yield a more easily controlled expansion than the more natural interpolation of the sum-if indeed this latter interpolation can be controlled.

8.B. Construction of $[\mathscr{A}]_{T}$

$[\mathscr{A}]_{T}$ is constructed inductively through the linear ordered expressions $[\mathscr{A}]_{T,(i, x)}$.

$$
\begin{aligned}
{[\mathscr{A}]_{T,(1,1)} } & =[\mathscr{A}] \\
{[\mathscr{A}]_{T,(i, x)} } & \rightarrow[\mathscr{A}]_{T} .
\end{aligned}
$$

Any single of these terms has the form

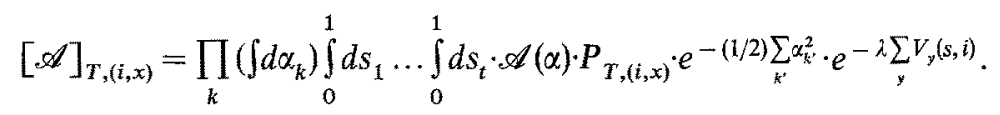

$P$ and $V$ are functions of the $\alpha$ 's and the s's. $t$ is the number of $(j, y)$ with $(j, y)<(i, x)$, and such that $T$ does not map $(j, y)$ into $N$. In Section 8.C, we will define, associated to $V_{x}(s, i)$, a $\hat{V}_{x}(s, i)$. All of the $V$ 's and $\hat{V}$ 's are homogeneous fourth degree polynomials in the $\alpha$ 's. We write $(i, x)_{+}$for the term succeeding $(i, x)$ in the (8.A.1) ordering.

If $T:(i, x) \rightarrow N$ then $[\mathscr{A}]_{T,(i, x)+}$ is derived from $[\mathscr{A}]_{T,(i, x)}$ by replacing the single term $V_{x}(s, i)$ in the exponent in (8.B.2) by $\hat{V}_{x}(s, i)=V_{x}(s, i+1)$. The interaction at a single lattice site is modified!

Otherwise, an integral $\int_{0}^{1} d s_{t+1}$ is added to the expression (8.B.2), $V_{x}(s, i)$ is replaced by

$$
V_{x}(s, i+1)=s_{t+1} V_{x}(s, i)+\left(1-s_{t+1}\right) \hat{V}_{x}(s, i)
$$

(again modification at a single lattice site), and $P_{T,(i, x)}$ is multiplied by the monomial term in $\frac{d}{d s_{t+1}}\left(-\lambda V_{x}(s, i+1)\right)$ that is specified by $T((i, x))$.

Given the interpolated interactions defined in the next section, the procedure of this section is a straightforward site by site interpolation - the notation is complicated.

\section{C. Interpolation of the Interaction}

If $S$ is a subset of the $k$ 's, we write

$$
\phi(S, x)=\sum_{k \in S} \alpha_{k}\left(\frac{1}{\sqrt{-\Delta}} \psi_{k}\right)(x) .
$$

We also write

$$
W(S, x)=(\Delta \phi(S, x))^{4} .
$$

In the next section we will associate to the term $T$, at the $(i, x)$ step, a partition of all the variables into sets $\left\{O^{(i, x)}, I_{j}^{(i, x)}\right\}$. Here we have suppressed the $T$ label in 
the notation, and $j$ varies over a fixed range $1 \leqq j \leqq j_{0}(i, x, T)$. (In the usual cluster expansions there is a division into only two sets, interior and exterior variables.)

$V_{x}(s, i)$ is a linear combination of terms $W(S, x)$ (for various subsets $S$ ). $\hat{V}_{x}(s, i)$ is obtained from $V_{x}(s, i)$ by the replacement

$$
W(S, x) \rightarrow W\left(S \cap O^{(i, x)}, x\right)+\sum_{j} W\left(S \cap I_{j}^{(i, x)}, x\right) .
$$

This is virtually the same way E's are interpolated in [2].

\section{D. Higher Structure}

We associate a graph to the term $T$ at the step $(i, x)$. The vertices are

a) the distinguished $\alpha_{k}$

b) those $y$ such that for some $j$

$(j, y)<(i, x)$ and $T((j, y)) \neq N$

c) those $\alpha_{k}$ that are in the image of $T$ for

$(j, y)<(i, x)$.

The vertices are thus the distinguished variables, $\alpha$ 's that have been differentiated down, and the sites at which they have been differentiated down. Each vertex corresponding to an $\alpha_{k}$ that is not distinguished is given a weight $w\left(\alpha_{k}\right)$, the number of times the $\alpha_{k}$ is differentiated down, counting multiplicity. The weight of $\alpha_{k}$ is thus the degree of $\alpha_{k}$ in $P_{T,(i, x)}$. There is a bond connecting an $\alpha_{k}$ and $y$ if for some $j,(j, y)<(i, x)$, and $\alpha_{k}$ is in the image of $(j, y)$ under $T$. Our graph clearly represents the connectivity properties of the interaction terms differentiated down. It will consist of a number of connected components; each connected component must contain some distinguished variable (for a term $T$ such that $[\mathscr{A}]_{T} \neq 0$ ).

The union of the $\alpha$ 's appearing in the graph are the "interior variables". These will be $\bigcup_{j} I_{j}^{(i, x)}$. The complementary set is the set of "exterior variables" $O^{(i, x)}$. We will have to work harder to define the subsets into which the interior variables are divided. We will sometimes mean the interior and exterior variables associated to a term $T$ to be

$$
\lim _{(i, x)} \bigcup_{j} I_{j}^{(i, x)}
$$

and

$$
\lim _{(i, x)} O^{(i, x)}
$$

associated to $T$ (the interior and exterior variables associated to sufficiently large $(i, x))$.

A subset of $\alpha$ 's in our graph is a "tadpole subset" if

a) The subgraph containing as vertices these $\alpha$ 's and all the vertices corresponding to $x$ 's connected by bonds to these $\alpha$ 's in the original graph, and whose bonds are inherited from the original graph, is connected.

b) None of the $\alpha$ 's is distinguished.

c) The sum of the weights of all the $\alpha$ 's is equal to $3(\bmod 4)$.

d) There is only one $\alpha_{k}$ not in the tadpole subset, call it $\alpha_{p}$, that is joined by a 
bond in the original graph to one of the $x$ in the subgraph of a). For all $\alpha_{k}$ in the tadpole subset one has

$$
r_{k}<r_{p}
$$

The partition into $I_{j}^{(i, x)}$ of the interior variables is the coarsest partition of the interior variables such that any tadpole subset is a union of some $I_{j}^{(i, x)}$.

\section{E. Basic Result}

At the end of the iterative construction for $[\mathscr{A}]_{T}$ one has an expression of the form $K_{T}(\mathscr{A}) Z^{T^{c}}$ for $[\mathscr{A}]_{T}$. Here $K_{T}(\mathscr{A})$ is an expression of the form (8.B.2), where the sums and products are restricted to $\alpha_{k}$ in the set of interior variables, and the $V$ 's also depend only on interior variables. $Z^{T^{c}}$ is the expression for $Z((8.2)$ with $\mathscr{A}=1)$, with products and sums restricted to exterior variables, and $(\Delta \phi)_{x}^{4}$ containing only contributions from the exterior variables. We define $|T|$ as the total number of interior variables in the term $T$.

Main Theorem. There is an $M_{0}$, such that given any $M \geqq M_{0}$ and any $c>0$, there is a $\lambda_{0}(M, p, c)$, such that

$$
\sum_{T}\left|K_{T}(\mathscr{A})\right| e^{c|T|} \leqq c_{1}(\mathscr{A}),
$$

for $0 \leqq \lambda \leqq \lambda_{0}$.

\section{F. A Few Remarks About Clustering}

There are standard techniques used to extract clustering properties of correlation functions from cluster expansions. These are usually, but not always, estimates for exponential fall-off with respect to spatial separation of arguments. In our case we expect power law fall-off. Our main interest is extending the present program to include more complex models such as the dipole gas, rather than extracting complete results for the present model. In any case, for the model $\lambda\left(|\Delta|^{\alpha} \phi\right)^{2 s}, \alpha>1 / 2$, clustering properties are more easily obtained along the lines in [8]. We restrict our remarks to the two point correlation function.

As the fields $\phi(x)$ are not our basic variables, but rather the quantities $\alpha_{k}$, it is perhaps not clear even what clustering properties it is natural to seek. We will state our results for the model $\lambda\left(|\Delta|^{\alpha} \phi\right)^{4}, \alpha>1 / 2$, and consider correlations of $|\Delta|^{\alpha} \phi$, probably the most interesting quantities to treat. We thus study

$$
\left\langle|\Delta|^{\alpha} \phi(x)|\Delta|^{\alpha} \phi(y)\right\rangle \text {. }
$$

We expect, for $\lambda$ small enough, that this will be bounded $\sim \frac{1}{|x-y|^{1+4 \alpha}}$. In Part 2, Appendix B, we will derive the weaker result:

Clustering Theorem. For any $\gamma<3$, there is a $\lambda_{y}$ such that for $0 \leqq \lambda \leqq \lambda_{\gamma}$

$$
\left|\left\langle|\Delta|^{\alpha} \phi(x)|\Delta|^{\alpha} \phi(y)\right\rangle\right| \leqq c_{\gamma} \frac{1}{|x-y|^{\gamma}} .
$$

As we stated above we expect that 3 may be replaced by $1+4 \alpha$ in the statement of the theorem. 
To give some indication of the proof (and our difficulty deriving the correct power), we substitute (5.1) into (8.F.1).

$$
\sum_{k} \sum_{k^{\prime}}\left(\frac{|\Delta|^{\alpha}}{\sqrt{-\Delta}} \psi_{k}(x)\right)\left(\frac{|\Delta|^{\alpha}}{\sqrt{-\Delta}} \psi_{k^{\prime}}(y)\right)\left\langle\alpha_{k} \alpha_{k^{\prime}}\right\rangle
$$

The lowest order contribution of the cluster expansion will thus yield

$$
\sum_{k}\left(\frac{|\Delta|^{\alpha}}{\sqrt{-\Delta}} \psi_{k}(x)\right)\left(\frac{|\Delta|^{\alpha}}{\sqrt{-\Delta}} \psi_{k}(y)\right) c_{k},
$$

with the $c_{k}$ uniformly bounded. Roughly $\frac{|\Delta|^{\alpha}}{\sqrt{-\Delta}} \psi_{k}(x)$ lives on a region of diameter $p^{r_{k}}$ and therein has a dimensional bound $\sim\left(p^{r_{k}}\right)^{-1 / 2-2 \alpha}$ (by estimates as in Sect. 7). The sum in (8.F.4) is dominated by terms with $p^{r_{k}} \sim|x-y|$. This suggests a $\frac{1}{|x-y|^{1+4 \alpha}}$ law. However it is the last term in (7.4)-rather the generalization of this term to the $\alpha \neq 1$ case - that causes us difficulties. In the convergence proof in Part 2 some slight juggling is also necessary to handle this term. Here the problem is that this last term in the generalization of (7.4) will have a uniform bound $\sim\left(p^{r_{k}}\right)^{-3 / 2}$. To improve our bound we would have to deal more effectively with regions where $\hat{d}$ is small. If one can use estimates wherein $\hat{d}$ is effectively $\sim L_{\alpha}$, then one would get the improved clustering theorem, 3 replaced by $1+4 \alpha$. We do not pursue this in the present work.

As is usual in a cluster expansion one needs estimates for $Z^{T^{c}} / Z$ (see (8.1)). These may be derived by the method of [4], now standard.

\section{References}

1. Benfatto, G., Cassandro, M., Gallavotti, G., Nicolo, F., Olivieri, E., Presutti, E., Scacciatelli, E. : Commun. Math. Phys. 59, 143-166 (1978)

2. Brydges, D., Federbush, P.: Commun. Math. Phys. 73, 197-246 (1980)

3. Gawedzki, K., Kupiainen, A. A Rigorous Block Spin Approach to Massless Lattice Theories, Harvard Preprint

4. Glimm, J., Jaffe, A., Spencer, T. : The Particle Structure of the Weakly Coupled $\mathbf{P}(\phi)_{2}$ Model and Other Applications. In: Lecture Notes in Physics, Vol. 25 Velo, G., Wightman A. (eds.) Berlin, Heidelberg New York: Springer 1973

5. Fröhlich, J., Spencer, T. : On the Statistical Mechanics of Classical Coulomb and Dipole Gases, Preprint

6. Gawedzki, K. : Long distance behavior for a critical hierarchical model, Preprint

7. Bricmont, J., Fontaine, J. R., Lebowitz, J. L., Spencer, T. : Lattice systems with a continuous symmetry, I, II. Preprint

8. Malyshev, V. A., Tirozzi, B.: Renormalization group convergence for small perturbations of Gaussian random fields with slowly decaying correlations, Preprint

Communicated by A. Jaffe

Received October 7, 1980; in revised form January 15, 1981 